\title{
The European X-ray Free-Electron Laser: toward an ultra-bright, high repetition-rate $x$-ray source
}

\author{
M. Altarelli \\ European XFEL GmbH, Albert-Einstein-Ring 18, 22761 Hamburg, Germany \\ (Received 31 January 2015; revised 29 April 2015; accepted 20 May 2015)
}

\begin{abstract}
The status of the European X-ray Free-Electron Laser (European XFEL), under construction near Hamburg, Germany, is described. The start of operations of the LCLS at SLAC and of SACLA in Japan has already produced impressive scientific results. The European XFEL facility is powered by a $17.5 \mathrm{GeV}$ superconducting linear accelerator that, compared to these two operating facilities, will generate two orders of magnitude more pulses per second, up to 27000. It can therefore support modes of operation switching the beam up to 30 times per second among three different experiments, providing each of them with thousands of pulses per second. The scientific possibilities opened up by these capabilities are briefly described, together with the current instrumental developments (in optics, detectors, lasers, etc.) that are necessary to implement this program.
\end{abstract}

Keywords: femtosecond lasers and applications; free-electron lasers and applications; repetition frequency technology

\section{Introduction}

The purpose of the present article is to outline the main features of the European X-ray Free-Electron Laser (European XFEL) Facility and its expected impact in some areas of scientific research. In Section 2, the main parameters of this X-ray source are briefly reviewed, together with a description of the present status and the forthcoming steps of the construction project; the repetition rate and the time structure of the pulse trains, together with the recent developments in the detector and instrumentation area are presented. In Section 3, some ideas about the possible operation modes and their impact on scientific experiments are discussed with a few examples.

\section{The European X-ray Free-Electron Laser Facility}

There are at present two operating hard x-ray free-electron lasers worldwide, one in the USA (the Linac Coherent Light Source, LCLS, in Stanford, California ${ }^{[1]}$ ), which delivered the first beam at $0.15 \mathrm{~nm}$ in April 2009; and one in Japan (the SPring-8 Angstrom Compact Laser, SACLA ${ }^{[2]}$ ), operational since June 2011; there are a further three projects in the construction phase, one in Switzerland (Swiss FEL) at the Paul Scherrer Institute ${ }^{[3]}$, one in South Korea, at

Correspondence to: M. Altarelli, European XFEL GmbH, Notkestr. 85, 22607 Hamburg, Germany. Email: massimo.altarelli@xfel.eu the Pohang Accelerator Laboratory ${ }^{[4]}$, and the European XFEL near Hamburg in Germany ${ }^{[5]}$. All of them target the production of wavelengths of the order of or smaller than $0.1 \mathrm{~nm}$, suitable for experiments determining structural properties with atomic resolution. The European XFEL, which has been under construction since January 2009, is powered by a $1.7 \mathrm{~km}$ long superconducting linear accelerator, based on the TESLA technology already tested in the soft $\mathrm{X}$-ray FLASH facility at DESY ${ }^{[6]}$. The adoption of the superconducting technology allows the production of up to 27000 pulses/s, to be compared with the typical $\sim 100 \mathrm{pulses} / \mathrm{s}$ of normal-conducting linac FELs. In Table 1 the main design parameters of the European XFEL are compared with those of the two operating facilities.

The time distribution of the 27000 pulses/s is dictated by the properties of the RF system and by the need to limit the cryogenic power requirements. The bunches will be delivered (see Figure 1) within electron bunch trains, each train $600 \mu \mathrm{s}$ long, and containing up to 2700 bunches; within the basic $10 \mathrm{~Hz}$ repetition rate of the RF system, successive trains are separated by nearly $100 \mathrm{~ms}$, whereas, inside each train, consecutive bunches are spaced by $\sim 222 \mathrm{~ns}$, corresponding to an effective repetition rate, during each train, of $\sim 4.5 \mathrm{MHz}$.

After reaching their final energy at the end of the linac, electron bunches can be directed in either of two electron beamlines, and pass through long undulators, as schematically illustrated in Figure 2. 


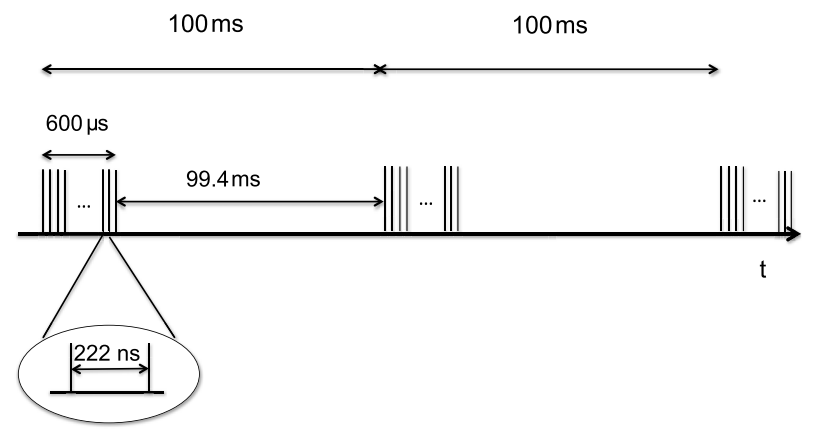

Figure 1. Schematic representation of the time sequence of both the electron bunches and the x-ray pulses at the European XFEL.

Table 1. Basic parameters of the European XFEL.

\begin{tabular}{lccc}
\hline Facility & $\begin{array}{c}\text { LCLS } \\
\text { (USA) }\end{array}$ & $\begin{array}{c}\text { SACLA } \\
\text { (Japan) }\end{array}$ & $\begin{array}{c}\text { European } \\
\text { XFEL (SASE1 } \\
\text { undulator) }\end{array}$ \\
\hline Max. electron energy (GeV) & 14.3 & 8.0 & 17.5 \\
Min. photon wavelength (nm) & 0.15 & 0.06 & $<0.05$ \\
Photons/pulse & $\sim 10^{12}$ & $2 \times 10^{11}$ & $\sim 10^{12}$ \\
Peak brilliance & $\sim 8 \times 10^{32}$ & $\sim 10^{33}$ & $5 \times 10^{33}$ \\
Average brilliance & $2 \times 10^{21}$ & $3 \times 10^{20}$ & $\sim 10^{25}$ \\
Pulses/second & 120 & 60 & 27000 \\
Date of first beam & 2009 & 2011 & 2016 \\
\hline
\end{tabular}

Basic parameters of the European XFEL in comparison with those of the two operating hard $\mathrm{x}$-rays FEL projects; brilliances are expressed in photons $/ \mathrm{s} / \mathrm{mrad}^{2} / \mathrm{mm}^{2} / 0.1 \% \mathrm{BW}$.

In the undulators, the electron bunches will produce photon pulses by the 'SASE' process ${ }^{[7]}$; the time structure of the photon pulses is a faithful reproduction of the bunch time structure depicted in Figure 1. The first beamline contains a hard $\mathrm{x}$-ray undulator (SASE1), generating photons tunable between 8.4 and $\sim 30 \mathrm{keV}$ (at $17.5 \mathrm{GeV}$ electron energy: softer x-ray radiation is of course obtained if the electron energy is reduced, according to the $\gamma^{2}$ undulator law), and a soft x-ray undulator (SASE3), which can under some circumstances (see Section 3) make use of the 'spent' beam resulting from saturation of SASE1 to generate soft $\mathrm{x}$-rays in the 0.78 to $3.1 \mathrm{keV}$ range (at $17.5 \mathrm{GeV}$ ). The second beamline (upper branch in Figure 2) contains a second hard x-ray undulator (SASE2), identical to SASE1, and two initially empty tunnels downstream, in which two further undulators can be located later, in future upgrades of the facility. The hard x-ray undulators consist of 35 segments, each $5 \mathrm{~m}$ long, with a period of $40 \mathrm{~mm}$; the soft $\mathrm{x}$-ray undulator SASE3 consists of 21 such segments, with a $68 \mathrm{~mm}^{\text {period }^{[8]}}$. $^{2}$

In the baseline design for the initial phase of the facility, each of the three installed SASE undulators will feed into two instruments; in principle a third one could be added.

The photon beams produced in the undulators are directed to the experimental hall through long optical transport systems; the SASE1 optical beamline is over $900 \mathrm{~m}$ long. The long drift distances ensure reduced optical load on mirrors and crystals, as well as sufficient separation in the $90 \mathrm{~m}$ wide experiment hall for the instruments fed by different undulators.

Altogether, the linear length of the facility is over $3 \mathrm{~km}$, starting within the DESY site with the electron gun and the injector, and ending with the experiment hall in the town of Schenefeld (Figure 3). The facility is completely underground, at a depth varying between 6 and $38 \mathrm{~m}$. The underground civil construction (tunnels, shafts, experiment hall) was completed in the summer of 2013. At present, mass

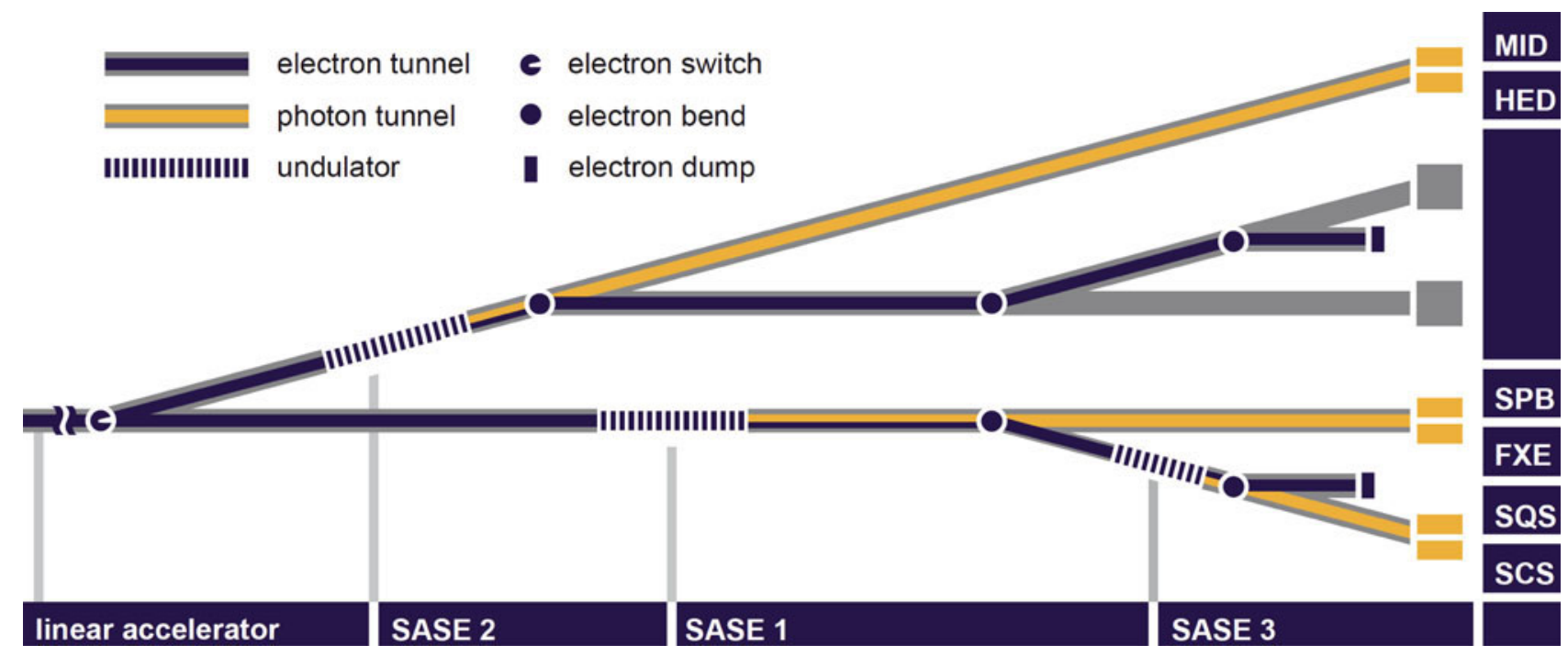

Figure 2. Schematic representation of the layout of the undulator and photon tunnels and of the instruments at the European XFEL. The linear accelerator is to the left of the figure. The SASE1, SASE2 and SASE3 undulator positions are visible; between the SASE1 and the SASE2 tunnels, the two additional undulator tunnels available for future developments are shown. Far right: the acronyms of the six baseline instruments are explained in Table 3. 


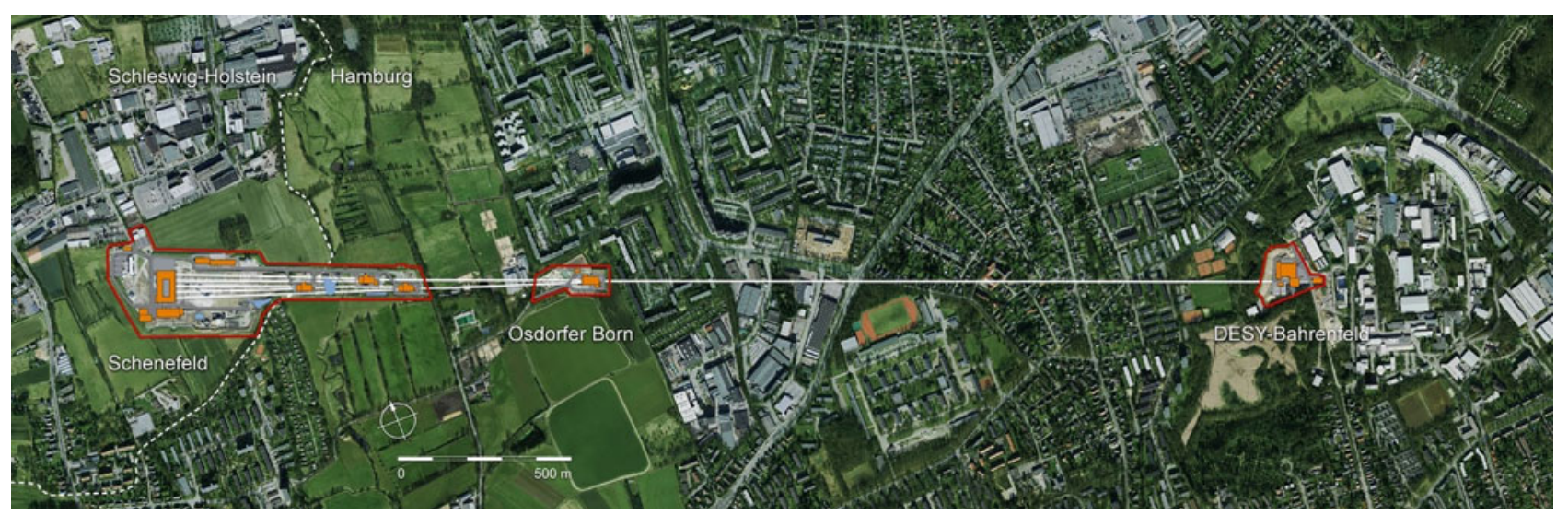

Figure 3. Layout of the European XFEL facility; the path of the underground tunnels is superimposed on a map of the northwest part of Hamburg and the town of Schenefeld in Schleswig-Holstein.

Table 2. Target performances of detectors for the European XFEL.

\begin{tabular}{lccc}
\hline Detector parameters & AGIPD & LPD & DEPFET sensor with signal compression (DSSC) \\
\hline Energy range $(\mathrm{keV})$ & $3-13(25)$ & $5(1)-20(25)$ & $0.5-6(25)$ \\
Dynamic range (photons/pixel/pulse) & $10^{4} @ 12 \mathrm{keV}$ & $10^{5} @ 12 \mathrm{keV}$ & $6000 @ 1 \mathrm{keV}$ \\
Single photon sensitivity & yes & yes & yes \\
Number of intermediate stored images & $\sim 360$ & 512 & $\sim 800$ \\
Pixel size $\left(\mu \mathrm{m}^{2}\right)$ & $200 \times 200$ & $500 \times 500$ & $236 \times 236$ \\
\hline
\end{tabular}

Target performances of three MHz repetition-rate Megapixel position sensitive detectors under development for the European XFEL. Values in parentheses refer to range extension with reduced performance. The number of images (next to last row) refers to the number of images that can be stored out of a pulse train with $4.5 \mathrm{MHz}$ repetition rate.

manufacturing of accelerator, $x$-ray beamlines and instrument components is in progress. The installation of the gun was completed in late 2013, and its commissioning started in the last quarter of 2013. The injector installation will be completed in early summer 2015, with commissioning due to start in July 2015; the linac components installation started in 2014, with the aim of completing the assembly by mid 2016 . The installation of the undulators and of the x-ray beamtransport systems started in the final quarter of 2014, first with SASE1 and SASE3 and the related systems, and about one year later, by fall 2015, it will continue with SASE2 and the respective beamline. The installation of the hutches, of their infrastructure and safety systems will follow the same sequence, so that SASE1 instruments will be installed starting in early 2016 , in order to be ready for commissioning with a beam at the very end of 2016, followed by SASE3 and then SASE2.

One of the major challenges in the delivery of the instrumentation for the European XFEL is the handling and effective use of the very closely spaced pulses of each pulse train, separated by $\sim 222 \mathrm{~ns}$. This is a challenge for the optical and beam-transport components ${ }^{[9]}$ (as the SASE photon pulses are of the order of $\sim 1 \mathrm{~mJ}$ each, during the $600 \mu \mathrm{s}$ train the average power of the photon beam is several $\mathrm{kW}$ ), the diagnostics ${ }^{[10]}$, the optical lasers for pump-probe experiments $^{[11]}$, and for the detectors - in particular the
Megapixel [Mpx] two-dimensional (2D) imaging detectors, which should read-out and accumulate images at a $4.5 \mathrm{MHz}$ rate $^{[12]}$. An extensive instrumentation R\&D program was therefore launched at an early stage, in cooperation with laboratories all over Europe. The target parameters for thw three imaging Mpx detector developments are described in Table 2.

It is important to notice that smaller area prototypes of the adaptive gain integrated pixel detector (AGIPD) and large pixel detector (LPD) detectors have been tested at synchrotron sources, in order to demonstrate $\mathrm{MHz}$ image acquisition rates, with encouraging results.

The investment and technological hurdles in the detector development are especially challenging; however, the effort in instrumentation development is not limited to this area, nor to data acquisition and data treatment strategies: $\mathrm{x}$ ray optical elements, photon diagnostic and characterization tools, and burst-mode optical lasers for pump-probe experiments are equally essential areas for the success of the European XFEL facility.

Among the optical elements that are needed to transport the photons to the experiment hall, the system of deflecting and focusing mirrors is particularly challenging ${ }^{[9]}$. In the most extreme cases the specifications include a $2 \mathrm{~nm}$ peakto-valley profile error over an $80 \mathrm{~cm}$ long footprint on a mirror. Prototypes have been developed and tested, and very 
Table 3. Schematic description of the scientific applications of the six baseline instruments.

\begin{tabular}{|c|c|c|}
\hline \multirow{4}{*}{ Hard $x$-rays } & \multirow{2}{*}{ SASE1 } & $\begin{array}{l}S P B-S F X: \text { Single particles, clusters and biomolecules - serial femtosecond crystallography (User } \\
\text { Consortium): Structural determination of (mostly) biological objects }\end{array}$ \\
\hline & & $\begin{array}{l}\text { FXE: Femtosecond X-ray experiments: time-resolved investigation of ultrafast processes in solids, } \\
\text { liquids or gases }\end{array}$ \\
\hline & \multirow{2}{*}{ SASE2 } & $\begin{array}{l}\text { MID: Materials imaging and dynamics: structure and dynamics determination of disordered } \\
\text { systems by coherent scattering and photon correlation spectroscopy }\end{array}$ \\
\hline & & $\begin{array}{l}\text { HED: High energy-density matter: matter under extreme conditions, e.g., in very high fields, high } \\
\text { temperature and pressures }\end{array}$ \\
\hline \multirow{2}{*}{ Soft $x$-rays } & \multirow{2}{*}{ SASE3 } & $\begin{array}{l}\text { SQS: Small quantum systems: investigation of atoms, ions, molecules and clusters in intense fields, } \\
\text { nonlinear } x \text {-ray optical phenomena }\end{array}$ \\
\hline & & $\begin{array}{l}\text { SCS: Soft x-ray coherent scattering and spectroscopy: electronic and atomic structure, dynamics } \\
\text { of nano-systems and non-reproducible biological systems with soft } x \text {-rays }\end{array}$ \\
\hline
\end{tabular}

Schematic description of the scientific applications of the six baseline instruments, four operating in the hard, and two in the soft x-ray spectral region. In the top line, the SPB baseline instrument was combined with the SFX User Consortium experimental station, to constitute an instrument.

long lead times and buffers are foreseen to mitigate the risk on the timely delivery of mirrors.

Diagnostic devices ${ }^{[10]}$ for the photon beams aim at pulseto-pulse characterization of position, intensity, spectral and temporal information and polarization. After an intense development and design phase, the production of final diagnostic devices started in 2013, and continued through 2014, extending to many types of devices: gas-based intensity and position monitors, online photoemission spectrometers, diagnostic imagers, a special monochromator for undulator $\mathrm{K}$-tuning and commissioning, and $\mathrm{THz}$-streaking devices for temporal characterization.

The experience gained by the start of experimental activities on FELs worldwide has shown the importance of optical lasers as indispensable components of advanced FEL-based techniques for the study of ultrafast dynamics of matter. In the case of the European XFEL, in order to make optimal use of the high number of pulses, the time structure of the FEL source should be reproduced for the optical lasers $^{[11]}$. This has required an effort in the development of suitable burst-mode fs optical lasers, with suitable pulse energy and temporal jitter. The design is complete and has been critically reviewed; crucial components have been procured, assembled and tested; the complete assembly of the system and demonstration of the achievement of the specified parameters are still work in progress.

\section{Operation modes and scientific perspectives}

In the initial configuration, each of the three undulators will feed two instruments, as detailed in Table 3. The baseline program of the European XFEL will therefore provide six instruments to the science community. In addition, the User Consortia program will provide additional instrumentation and ancillary facilities; there are at present seven approved User Consortia; the most advanced is the SFX (serial femtosecond $\mathrm{X}$-ray crystallography), which will merge with and complement the SPB baseline instrument, enhancing the quality and quantity of the equipment available to structural biology users.

The undulator configuration of Figure 2 shows that the electron beam can take a path along the lower branch (SASE1-SASE3) or the upper branch (SASE2). It is foreseen that a very fast kicker magnet can direct one bunch train either to the upper or lower branch, or even split a bunch train in two, directing the first sequence of electron bunches to one branch and the second sequence to the other; in the latter case, however, a number of bunches of the order of a few tens will be lost, as the ramp of the switching magnet can be done only in a time one order of magnitude bigger than the time separation of consecutive bunches (222 ns); on the other hand, with 2700 bunches per train, the loss of 20 or 30 bunches does not compromise the delivery of over 10000 bunches/s to each branch.

Bunches taking the lower route will transit through the SASE1 and then the SASE3 undulator. Under some conditions, the same bunch can therefore produce hard x-ray FEL radiation in SASE1 and then FEL soft $\mathrm{x}$-ray radiation in SASE3. However, depending on electron energy, bunch charge, and gap setting (radiation wavelength) of SASE1, the energy spread after saturation of radiation at the end of SASE1 can vary ${ }^{[13]}$ between a few $\mathrm{MeV}$ and over $20 \mathrm{MeV}$. Typically, the induced energy spread is higher for small emittances (corresponding to small bunch charges). The larger the energy spread, the longer the minimum wavelength at which bunches 'spent' on SASE1 can achieve saturation of soft x-ray emission on SASE3. Therefore, under some circumstances, lasing in both SASE1 and SASE3 will not be possible. In such cases, lasing on SASE3 can be made possible, not only by obvious, but slow-moving changes of configurations (e.g., opening up the SASE1 gap completely); SASE1 lasing can also be prevented by a slight kick (of order $\sim 10 \mu \mathrm{rad}$ ) imparted on the electron beam by a fast kicker at the entrance of SASE1. Betatron oscillations in the SASE1 part of the trajectory prevent SASE1 lasing; the kick can be compensated at the exit of SASE1 by appropriate setting of a static steerer, e.g., a displaced quadrupole ${ }^{[14]}$. 
One can therefore envisage an operation mode in which a bunch train with up to 2700 bunches can be split into an initial sub-train proceeding through SASE1, and a second sub-train through SASE2. The first sub-train can be further split into a first part lasing in SASE1, and a second, slightly kicked part that lases in SASE3. With the loss of some tens of bunches, therefore, one can direct, for example, 10 pulse sub-trains per second, each with close to 900 pulses, to each of three instruments on SASE1, SASE3 and SASE2, respectively; this mode can therefore achieve simultaneous operation of three instruments with almost 9000 pulses/s. A pre-condition for this operating mode is of course that the three experiments have the same electron energy and bunch charge requirements.

Turning briefly to the scientific applications of the European XFEL, we notice that, when compared to other XFEL facilities, the unique features of the European XFEL are the high number of pulses/s, with the associated potential to serve several user groups simultaneously, and the very wide photon energy range (from $250 \mathrm{eV}$ to over $30 \mathrm{keV}$, within the first harmonic, by combined variation of the electron energy and the undulator gap setting).

The high number of pulses is eliciting very strong interest in the structural biology community ${ }^{[15]}$. While the success stories of nano-crystallography at LCLS are quite exciting, the improved repetition rate can, on the one hand, lead to a more efficient use of samples that are hard to purify and rather expensive; on the other hand, it can lead to the possibility of acquiring, in a reasonable time, sufficient frames to obtain a 'molecular movie' of a photochemical process - here the limiting factor is the detector intermediate storage possibilities, presently limited to $\sim 300$ to $\sim 500$ frames per pulse train, leading to $\sim 3000$ frames per second as a reasonable estimate.

Imaging methods based on reconstruction of structural features from diffraction patterns obtained with a transversely coherent source are of great interest not only for structural biology but also for condensed matter and materials science. Experiments with $\mathrm{x}$-ray FELs have already demonstrated the imaging of individual Au nanocrystals ${ }^{[16]}$, capturing the movie of deformations induced by the lattice response to a laser pump pulse, by successive snapshots at different laser-FEL delays. Other experiments on water drops, cooling while evaporating through vacuum ${ }^{[17]}$, showed either the diffuse scattering characteristic of liquids, or the superposition of broad and bright Bragg peaks from ice crystallites (with linear dimensions $12 \mathrm{~nm}$ or greater), superposed on the diffuse scattering.

The latter experiment probably ushers in a novel application of FELs to the physics of the liquid state and of the liquid-solid phase transition. (Very recently also an experiment on liquid $\mathrm{He}$ droplets at the LCLS has been reported $^{[18]}$.) As a matter of fact, all x-ray or neutron scattering experiments on liquids so far have been performed with acquisition times much longer than all the characteristic positional and orientational molecular relaxation times, delivering very interesting but time-averaged information, such as pair correlation or radial distribution functions. The ultra-short pulses of the x-ray FELs, on the other hand, are shorter than most such characteristic times and potentially deliver snapshots of the instantaneous configurations of the liquid, encoded in a speckle pattern (more precisely, projections of the instantaneous configurations on the detector plane, normal to the incoming beam direction). The short exposure time and the demonstrated capability of Bragg peak detection from crystalline nuclei, for example, can give a new handle on the phenomenon of nucleation at the liquidsolid transition, a topic of great importance for disciplines from materials science to atmospheric physics, with many open questions. Here the statistical study of the number, size and morphology (from indexing but also from direct imaging and study of the fringes) of crystallites in a large number of drops (thanks to the high number of pulses) are a definite advantage for our facility. As was pointed out recently ${ }^{[19]}$, the availability of high photon energies (beyond $20 \mathrm{keV}$ ) can play an important role for atomic scale probing of structures in crystallites a few $\mathrm{nm}$ in size.

More generally, all time-dependent problems that can be investigated by pump-probe experiments will benefit from the high number of pulses per second. For $\mathrm{x}$-ray probe experiments requiring significant $\mathrm{x}$-ray flux, such as $\mathrm{x}$-ray emission spectroscopies (XES), the integrated number of $\mathrm{x}$-ray photons is the bottleneck; consequently, it is highly advantageous to increase the number of $\mathrm{x}$-ray probe photons by a higher rep rate at equal number of photons per pulse. The FXE instrument group and the Data Acquisition group actually demonstrated ${ }^{[20]} \mathrm{MHz}$ rate pump-probe acquisition on synchrotron sources as early as 2012. It is very conservative to estimate an increase in effective $\mathrm{x}$-ray flux by a factor of $\sim 50$ over other FELs as a result of the number of pulses per second (to be combined with the effect of a higher number of photons per pulse, likely to occur in comparison to lower electron energy FELs), which will allow many new applications to photochemical and photobiological dynamics, where the low $\mathrm{S} / \mathrm{N}$ for achievable and/or physiological concentrations has so far been a very significant limitation

XES experiments can also be seen as special cases of 'photon-starved' experiments, such as those involving very dilute systems and small cross sections, in which the high repetition rate of the facility can play a decisive role; turning our attention away from time-resolved investigations, the high average brilliance of the European XFEL, resulting from the high number of pulses per unit time and reaching into the high $10^{24}$ figures (see Table 1), can presently surpass all other sources by at least two orders of magnitude. 
Consider for example non-resonant inelastic scattering with $\sim$ meV energy resolution. With proven techniques to improve the longitudinal coherence and the photon output (self-seeding mode of operation and undulator tapering ${ }^{[21]}$ ), the European XFEL used in an integrated mode can provide in excess of 100 times more incoming photons in the relevant bandwidth than other existing sources; and technical solutions for handling the power load on the monochromatoranalyzer system, based either on crystal optics or on nuclear scattering techniques, are feasible ${ }^{[22,23]}$. The extra factor of 100 in incoming photon flux can make the difference between a next-to-impossible experiment $\left(10^{-2}\right.$ counts/s) and a feasible one (1 count/s); for example scattering by pair breaking across the whole Brillouin zone in high-Tc superconductors $^{[24]}$ could become a valuable complement to photoemission for the study of quasiparticle spectral densities.

Furthermore, 100-fold speeding up of data accumulation at different transferred energies and momenta can greatly extend the applicability of the procedure to extract subfs time responses from the Kramers-Kronig and Green function approach proposed by Abbamonte ${ }^{[25,26]}$.

\section{Outlook and conclusions}

User operation of the European XFEL Facility is expected to start in 2017. Although early users will be confonted with a machine and instrumentation not yet delivering all the parameters (repetition rate, rapid beam switching between experiments, pump laser synchronization, stability...) in an optimal way, they will begin to harvest the scientific promise of the European XFEL. As we have tried to explain in this article, the unique features of the facility (high repetition rate, the flexibility provided by the high electron energy and by the different undulators) will open the way to novel experiments in a variety of scientific fields, in which simultaneous access to atomic-scales of space and time is essential. A further important feature of the European XFEL is the possibility of expansions and upgrades, with two available empty undulator tunnels downstream of the SASE2 undulator; and with plenty of space in the long undulator and beamline tunnels, as well as in the experiment hall, for additional equipment and instrumentation. In the rapidly developing technical and scientific environment of accelerator-based light sources, this is a further guarantee of a durable presence at the very forefront of science for $\mathrm{x}$-ray free-electron lasers.

\section{Acknowledgements}

The whole team at the European XFEL and DESY should receive credit for the work presented here. Illuminating discussions with G. Geloni, A. P. Mancuso, M. Kuster, H. Sinn and Th. Tschentscher (European XFEL), and with G. Monaco (University of Trento, Italy) are gratefully acknowledged.

\section{References}

1. P. Emma, R. Akre, J. Arthur, R. Bionta, C. Bostedt, J. Bozek, A. Brachmann, P. Bucksbaum, R. Coffee, F.-J. Decker, Y. Ding, D. Dowell, S. Edstrom, A. Fisher, J. Frisch, S. Gilevich, J. Hastings, G. Hays, Ph. Hering, Z. Huang, R. Iverson, H. Loos, M. Messerschmidt, A. Miahnahri, S. Moeller, H.-D. Nuhn, G. Pile, D. Ratner, J. Rzepiela, D. Schultz, T. Smith, P. Stefan, H. Tompkins, J. Turner, J. Welch, W. White, J. Wu, G. Yocky, and J. Galayda, Nat. Photon. 4, 641 (2010).

2. T. Ishikawa, H. Aoyagi, T. Asaka, Y. Asano, N. Azumi, T. Bizen, H. Ego, K. Fukami, T. Fukui, Y. Furukawa, S. Goto, H. Hanaki, T. Hara, T. Hasegawa, T. Hatsui, A. Higashiya, T. Hirono, N. Hosoda, M. Ishii, T. Inagaki, Y. Inubushi, T. Itoga, Y. Joti, M. Kago, T. Kameshima, H. Kimura, Y. Kirihara, A. Kiyomichi, T. Kobayashi, C. Kondo, T. Kudo, H. Maesaka, X. M. Maréchal, T. Masuda, S. Matsubara, T. Matsumoto, T. Matsushita, S. Matsui, M. Nagasono, N. Nariyama, H. Ohashi, T. Ohata, T. Ohshima, S. Ono, Y. Otake, C. Saji, T. Sakurai, T. Sato, K. Sawada, T. Seike, K. Shirasawa, T. Sugimoto, S. Suzuki, S. Takahashi, H. Takebe, K. Takeshita, K. Tamasaku, H. Tanaka, R. Tanaka, T. Tanaka, T. Togashi, K. Togawa, A. Tokuhisa, H. Tomizawa, K. Tono, S. Wu, M. Yabashi, M. Yamaga, A. Yamashita, K. Yanagida, C. Zhang, T. Shintake, H. Kitamura, and N. Kumagai, Nat. Photon. 6, 540 (2012).

3. R. Ganter, (Ed.) PSI Report 10-06 (2010); see also P. Oberta, U. Flechsig, and R. Abela, Proc. SPIE 8078, 807805 (2011).

4. J.-H. Han, H.-S. Kang, and I. S. Ko, in Proceedings of IPAC2012 New Orleans, Louisiana, USA, p. 1735 (2012).

5. M. Altarelli, R. Brinkmann, M. Chergui, W. Decking, B. Dobson, S. Düsterer, G. Grübel, W. Graeff, H. Graafsma, J. Hajdu, J. Marangos, J. Pflüger, H. Redlin, D. Riley, I. Robinson, J. Rossbach, A. Schwarz, K. Tiedtke, T. Tschentscher, I. Vartaniants, H. Wabnitz, H. Weise, R. Wichmann, K. Witte, A. Wolf, M. Wulff, and M. Yurkov, (eds) in XFEL: The European X-Ray Free-Electron Laser Technical Design Report, http://xfel.desy.de/technical_inform ation/tdr/tdr/ (2006).

6. See e.g., http://flash.desy.de.

7. E. L. Saldin, E. V. Schneidmiller, and M. V. Yurkov, in The Physics of Free-Electron Lasers (Springer Verlag, Berlin, 1999).

8. J. Pflüger, M. Bagha-Shanjani, A. Beckmann, K. H. Berndgen, P. Biermordt, G. Deron, U. Englisch, S. Karabekyan, B. Ketenoğlu, M. Knoll, Y. Li, F. Wolff-Fabris, and M. Yakopov, in Proceedings of FEL13, 367 (2014), http://epaper.kek.jp/FE L2013/.

9. H. Sinn, M. Dommach, X. Dong, D. La Civita, L. Samoylova, R. Villanueva, and F. Yang, http://www.xfel.eu/document s/technical_documents/, doi:10.3204/XFEL.EU/TR-2012-006 (December 2012).

10. J. Gruenert, J. Buck, F. Dietrich, W. Freund, A. Koch, and M. Planas, in Proceedings of Free Electron Laser Conference 2014 (2014), http://www.fel2014.ch/prepress/FEL2014/.

11. L. Wissmann, M. Emons, M. Kellert, K. Kruse, M. Lederer, G. Palmer, M. Pergament, G. Priebe, J. Wang, and U. Wegner, in Proceedings of Free Electron Laser Conference 2014 (2014), http://www.fel2014.ch/prepress/FEL2014/.

12. J. Sztuk-Dambietz, A. Koch, M. Kuster, and M. Turcato, Proc. SPIE 8778, 87780U (2013). 
13. E. A. Schneidmiller and M. V. Yurkov, Technical Report DESY 11-152; XFEL.EU TR-2011-006 (2011); bib-pubdb1. desy.de/record/92341.

14. R. Brinkmann, E. A. Schneidmiller, and M. V. Yurkov, Nucl. Instrum. Methods Phys. Res. A 616, 81 (2010).

15. M. Mitchell Waldrop, Nature 505, 604 (2014).

16. J. N. Clark, L. Beitra, G. Xiong, A. Higginbotham, D. M. Fritz, H. T. Lemke, D. Zhu, M. Chollet, G. J. Williams, M. Messerschmidt, B. Abbey, R. J. Harder, A. M. Korsunsky, J. S. Wark, and I. K. Robinson, Science 341, 51 (2013).

17. J. A. Sellberg, C. Huang, T. A. McQueen, N. D. Loh, H. Laksmono, D. Schlesinger, R. G. Sierra, D. Nordlund, C. Y. Hampton, D. Starodub, D. P. DePonte, M. Beye, C. Chen, A. V. Martin, A. Barty, K. T. Wikfeldt, T. M. Weiss, C. Caronna, J. Feldkamp, L. B. Skinner, M. M. Seibert, M. Messerschmidt, G. J. Williams, S. Boutet, L. G. M. Pettersson, M. J. Bogan, and A. Nilsson, Nature 510, 381 (2014).

18. L. F. Gomez, K. R. Ferguson, J. P. Cryan, C. Bacellar, R. M. P. Tanyag, C. Jones, S. Schorb, D. Anielski, A. Belkacem, C. Bernando, R. Boll, J. Bozek, S. Carron, G. Chen, T. Delmas, L. Englert, S. W. Epp, B. Erk, L. Foucar, R. Hartmann, A. Hexemer, M. Huth, J. Kwok, S. R. Leone, J. H. S. Ma, F. R. N. C. Maia, E. Malmerberg, S. Marchesini, D. M. Neumark, B.
Poon, J. Prell, D. Rolles, B. Rudek, A. Rudenko, M. Seifrid, K. R. Siefermann, F. P. Sturm, M. Swiggers, J. Ullrich, F. Weise, P. Zwart, C. Bostedt, O. Gessner, and A. F. Vilesov, Science 345, 906 (2014).

19. J. Gulden, O. M. Yefanov, E. Weckert, and I. A. Vartanyants, AIP Conf. Proc. 1365, 42 (2011).

20. K. Haldrup, G. Vankó, W. Gawelda, A. Galler, G. Doumy, A. M. March, E. P. Kanter, A. Bordage, A. Dohn, T. B. van Driel, K. S. Kjær, H. T. Lemke, S. E. Canton, J. Uhlig, V. Sundström, L. Young, S. H. Southworth, M. M. Nielsen, and C. Bressler, J. Phys. Chem. A 116, 9878 (2012).

21. S. Serkez, V. Kocharyan, E. Saldin, I. Zagorodnov, G. Geloni, and O. Yefanov, in Proceedings of FEL13, 574 (2014), http://epaper.kek.jp/FEL2013/.

22. H. Sinn, private communication.

23. G. Monaco, private communication.

24. P. Johansson and M. Altarelli, Phys. Rev. B 53, 8726 (1995).

25. P. Abbamonte, K. D. Finkelstein, M. D. Collins, and S. M. Gruner, Phys. Rev. Lett. 92, 237401 (2004).

26. P. Abbamonte, T. Graber, J. P. Reed, S. Smadici, C.-L. Yeh, A. Shukla, J.-P. Rueff, and W. Ku, Proc. Natl Acad. Sci. USA 105, 12159 (2008). 